\title{
Über den nervösen Ursprung der telangiektatischen und anämischen Naevi.
}

\author{
Von \\ A. Buschke. \\ (Aus der dermatologischen Abteilung des Rudolf-Virchow-Krankenhauses in \\ Berlin.)
}

Mit I Textabbildung.

Über die von Voerner unter dem Namen „Naevus anaemicus“ zuerst in diesem Archiv im Jahre 1906 berichtete Affektion ist seitdem aus meiner Klinik von W. Fischer, Bruner und von Stein aus Jadassohns Klinik das Wichtigste bezüglich der tatsächlichen. Befunde und das Mutmaßliche der Entstehungsweise berichtet worden. Ich will aus diesen Mitteilungen in aller Kürze ganz summarisch das referieren, was zum Verständnis der Affektion und zur Erweiterung der bezüglich der telangiektatischen Naevi von mir hier aufzustellenden Hypothese beiträgt. Es handelt sich um angeborene Anomalien, besonders an der Haut des Rumpfes und des Halses, seltener der Extremitäten, von meist scharfer, aber unregelmäßiger Begrenzung, in der Größe schwankend etwa von dem Umfange eines Markstückes bis zu Riesennaevis, welche große Strecken des Rumpfes überziehen können. Das Charakteristische der Affektion ist, daß die Hautfarbe blaß ist gegenüber der zartroten oder bläulichen Färbung der umgebenden Haut. Im übrigen ist die Oberfläche der Haut unverändert, atrophisehe Veränderungen bestehen nicht. Weitere Untersuchungen zeigen, daß die Blässe nicht dureh Pigmentmangel entsteht, sondern durch Blutleere der Haut. Hervorgehoben sei, daß gelegentlich im Naevus anaemicus vereinzelte Partien sich finden, in deren Bereich die Haut eine mehr der Norm sich nähernde Blutfüllung zeigt. Irgendwelche sonstigen Anomalien bezüglich der Sensibilität, der Schweißsekretion waren nicht zu konstatieren. Die histologische Untersuchung hat ein völlig negatives Resultat ergeben, besonders ist das Hautgefäßsystem normal entwickelt, und die Blutgefäße zeigen nur gegenüber der normalen. Haut bedeutend geringere Füllung. Hier sei gleich erwähnt, daß sowohl nach unseren wie den Beobachtungen der anderen Autoren die anaemischen Naevi nicht selten in geringerer oder sehr starker Ausdehnung mit telangiektatischen Naevis kombiniert sind. Da diese Kombinaton aber den wesentlichsten 
Grund zu der vorliegenden Mitteilung abgibt, komme ich später an der Hand unserer eigenen Beobachtungen auf diesen Punkt noch einmal zurück. Diese Naevi anaemici, welche uns zuerst als eine Rarität erschienen, sind, nachdem unsere Aufmerksamkeit einmal darauf gelenkt war, ziemlich häufig von uns beobachtet worden, wenn auch vorwiegend in der kleineren Form und besonders an der Vorderfläche; seltener an der Hinterfläche des Rumpfes, wobei erwähnt sein mag, daß gelegentlich um einen größeren Fleck sich kleinere gruppieren.

Bei der Abwesenheit jeglicher anatomischer Grundlage zur Erklärung dieser eigenartigen Erscheinung sind außer von Voerner selbst auch von Fischer, Bruner und Stein einige Versuche ausgeführt worden, um eine Klärung über die Frage der Entstehung dieser circumscripten angeborenen Hautanaemie herbeizuführen. Diese Versuche, auf welche ich im einzelnen hier nicht eingehen will, haben im wesentlichen Folgendes ergeben: Durch stauung ist es möglich, die Anämie der Haut völlig oder bis zu einem gewissen Grade aufzuheben. Nach Aufhören der Stauung versehwindet die Blutfülling wieder und macht der Anämie Platz. Durch mechanisches Reiben z. B. mit einer Bürste tritt der Naevus anaemicus meist sehr deutlich hervor, indem die umgebende Haut sich stark rötet, er aber blaß bleibt. Allerdings kann man gar nicht so selten beobachten, daß bei sehr starkem Reiben auch partieweise Rötungen im Bereiche des Naevus anaemic us auftreten. Wir haben vermittelst der Quarzlampe ein Erythem und Dermatitis erzeugt, welche sich nicht nennenswert von der der gesunden Umgebung unterschied. Stein beobachtete eine Maserneruption im Bereich des Naevus mit völlig normaler Blutfüllung. lm Gegensatz zu diesen Beobachtungen positiver Art verdient hervorgehoben zu werden, daß Voerner ebenso wie ich in mehreren Fällen feststellen konnte, daß bei diffusen Hautrötungen aus inneren, nervösen Gründen der Naevus vollkommen blaß blieb inmitten der stark geröteten Haut. Aus allen diesen and noch einigen anderen mit Morphiuminfiltration usw. besonders von Stein angestellten Versuchen scheint hervorzugehen, da $\beta$ alle diejenigen Reize, welche auf die Gefäßmuskulatur direkt wirken oder einfach mechanisch die Gefäße ausdehnen, eine Blutfüllung im Bereich des Naevus erzeugen können, während Reize, die durch Vermittlung des Nervensystems auf die Gefäße wirken, versagen, so daß hierdurch die zuerst von Fischer ausgesprochene Hypothese, da $B$ es sich um eine angeborene Anomalie der Gefäßnerven handele, diese Bildung am besten erklärt. Es würde sich dann um ein Fehlen oder eine Unterbildung der Vasodilatatoren handeln. Voerner hat sich sogar schon bemüht, diesen Gedanken in seiner zweiten Arbeit weiter auszubauen; ich werde nachher noch darauf zurückkommen. Soweit es sich also um einfache Naevi anaemici handelt, können wir diese Erklärung akzeptieren, wenngleich sie bis 
jetzt anatomisch noch nicht erwiesen ist. Nun habe ich aber bereits vorher erwähnt, daß diese Naevi sich gar nicht so selten mit kleineren telangiektatischen Naevis kombinieren. Besonders auffallend ist aber die Kombination von sehr großen Naevis anaemicis mit sehr ausgedehnten teleangiektatischen Naevis. Während wir in ersterem Falle noch von irgendwelchen Zufälligkeiten reden können, zwingt uns der letztere Befund dazu, für beide anatomisch und physiologisch so differenten Anomalien eine gemeinsame Ursache zu suchen, besonders wenn, wie in dem einen meiner Fälle anämische und telangiektatische Partien wahllos durcheinander geschüttelt sind. Es ergeben sich bei der Betrachtung solcher Fälle und dem. Versuch ihrer Erklärung allgemeine Gesichtspunkte für die Entstehung der Gefäßnaevi, welche von der bisherigeu Erklärung doch etwas abweichen und mir deshalb der Mitteilung wert erscheinen, dies hier umsomehr, als ja bekanntlich unser verehrter Jubilar zuerst auf eine bestimmte Form der GefäBnaevi, den Unnaschen, in der Mitte des Nackens lokalisierten Naevus, die Aufmerksamkeit gelenkt hat.

Fall 1. Es handelt sich um einen 23 Jahre alten Mann, der am 3. VI. 1912 zu uns mit einer Folliculitis barbae kam und als Nebenbefund einen Naevus anaemicus aufwies, der sich mit einem Naevus telangiektaticus kombinierte. Der Naevus anaemicus beginnt etwa in der Höhe des ersten. Brustwirbelfortsatzes und erstreckt sich ea. bis zum Schluß der Brustwirbelsäule, wesentlich auf der linken Seite des Rückens verlaufend und nur in seiner oberen Hälfte die Mittellinie nach rechts überschreitend, geht dann nach abwärts bis etwa zum dritten Lendenwirbel und ist hier in seiner größeren Ausdehnung links neben dem Lendenwirbel lokalisiert; rechts reicht er etwa bis zu einer Senkrechten, die vom unteren Scapularwinkel nach abwärts gezogen wird, links etwa bis zwei Querfinger breit von der hinteren Axillarlinie. Die Grenzen dieses Nacvus anaemieus sind scharf gegen die benachbarte gesunde Haut abgesetzt. Sie sind meistens unregclmäßig gezackt und nur links unten etwas regelmäßiger lineär gestaltet. Dieser Nacvus anaemicus ist nun verbunden mit einem ziemlich ausgedehnten strichförmigen telangiektatischen Naevus, welcher in der Hauptsache an der linken seite des Kopfes und des Rumpfes verläuft. Er beginnt ca. zwei Finger breit oberhalb des Scheitels, dicht denselben streifend, erstreckt sich dann nach links bis an die linke Ohrmuschel und zieht abwärts in zackiger Begrenzung bis an den oberen Rand des Sternocleidomastoideus. Dann geht er fast senkrecht abwärts bis an den hinteren Rand dieses Muskels wendet sich nochmals rechtwinklig nach innen und biegt dann etwa in Höhe des letzten Halswirbels senkrecht nach aufwärts um, überschreitet ein wenig dio Mittellinie, um dann am Scheitel zu endigen. Er ist dann durch gesunde Haut von dem etwas weiter abwärts liegenden Naevus anaemicus getrennt, aber dann, gewissermaßen in der Fortsetzung seiner Längsachse, finden sich etwa zwischen dem dritten und letzten Brustwirbeldorn in der Mitte drej telangiektatisehe Naevi, die durch anämische Partien getrennt sind, scharf aber unregelmäßig begrenzt, in ihren Ausläufern sich in das anämische Gebiet verijeren und in ihrer Fläche son kleinsten anämischen Bezirken durchsetzt sind. Der oberste dieser Herde vt etwa daumenballengroß, der daruntergelegene etwa ein Drittel kleiner und der unterste eben angedeutet. Außerdem finden sich an der lateralen Begrenzung der unteren linken Hälfte des Naevus anaemicus einige kleinste Telangiektasion. 
Noch bemerkenswerter ist der 2. Fall. Es handelt sich um einen 25 .Jahre alten Mann, der mit einer frischen Lues auf die Station kam. Abgesehen von dieser Affektion hatte der Patient ein ausgedehntes Feuermal, das sich, soweit er sich erinnert, nicht verändert hat. In seiner Familie (er hat 5 gesunde Geschwister) ist ähnliches nicht zur Beobaehtung gelangt. Den Befund dürfen wir als Veränderungen in Form von Naevus anaemicus und Naevus telangiektaticus und Übergänge zwischen beiden Veränderungen ansprechen. Befallen davon sind Gesicht, behaarter Kopf, Hals, rechter Ober- und Unterschenkel von oben nach unten, vorn und hinten bis zur Lendengegend. Am stärksten befallen ist der Rumpf an der Vorder- und Hinterfläche, die Schulterregion und die oberen Extremitäten. Normale Hautpartien wechseln mit telangiektatischen und anämischen ab. Die telangiektatischen Partien breiten sich vielfach in dicken Schichten aus und zeigen unregelmäßige Begrenzung. Dadurch, daß an einzelnen Stellen telangiektatische Haut in die normale hineingesprenkelt und in anaemische Stellen sozusagen hineingewachsen ist, bekommt die affizierte Haut ein ganz buntes Aussehen. Dabei ist die Färbung an den dickeren Stellen der Fpidermis an den Handtellem schwächer. Auf der rechten Brustseite dehnen sich reichliche telangiektatische Stellen einige Zentimeter vom seitlichen Sternalrand beginnend bis zur Schulterregion, nach dem Rücken über die Clavicula, von der Schulter abwärts bis zum Oberarm aus, in zerstreuten Partien am Oberarm beginnend, dann teils flächenhaft konfluierend, teils normale Haut zwischen sich lassend. Die Telangiektasien in der Nähe der rechten Brustwarze erstrecken sich nach der Bauchgegend abwärts in mehreren Abstufungen, die allmählich in die benachbarte Haut übergehen, teils an anaemische Partien dieser Region anstoßend. Auf der rechten Rückenhälfte verläuft, ausgehend von der Vert. prominens, ein etwa dreieckig gestaltetes anaemisches Feld bis zur Schultergelenksgegend und zum äußeren Rand der Scapula, nach abwärts bis etwa unter die Mitte der Scapula. Diese anämische Partie ist unregelmäßig umsäumt von telangiektatischen Stellen, die bis in das anaemische Feld hineinreichen. Die linke Brustseite wird vollständig von einem anämischen Feld bedeckt, das bis an den Hals reicht und auf das anämische Feld auf der linken Rückenseite übergreift. An der linken Schulterregion geht es allmählich in die gesunde Haut über. Nach abwärts schließen sich auf der linken Bauchseite unregelmäßige Telangiektasien an, die teils von gesunder Haut, teils von anämischer Haut durchsetzt sind. Die Bauchhaut rechts von der Linea alba sieht bis auf wenige minimale Telangiektasien normal aus. Die linksseitige telangiektatische Partie schließt ziemlich scharf an der Linea alba ab. Die linke Rückenhälfte weist bis etwas unterhalb der Seapula ein dreieckig gestaltetes, zum Teil mit gesunder Haut durchsetztes anämisches Feld auf, das ca. 3 Querfinger breit neben der Wirbelsäule abschließt, am Rande einige Telangiektasien aufweist, und nach außen bis fast an die hintere Axillarlinie reicht. Nach unten zu schließt sich bis an die Dorn fortsatzlinie nach innen, nach außen bis an die hintere Axillarlinie reichend, cine telangiektatische Partie an, die vielfach von gesunder Haut unterbrochen ist. Nach abwärts verliert sie sich in die Kreuzbeinregion. Über die Arme verlaufen flächenhafte Telangiektasien, wobei die Streckseiten weniger als die Beugeseiten befallen sind. Am rechten Arm reichen diese Veränderungen dorsalwärts ungefähr bis zu den Grundphalangen der Finger, palmarwärts ungefähr bis zu den Metakarpi. Der linke Arm ist im ganzen weniger befallen als der rechte. Hier treten die Telangiektasien mehr auf der Streckfläche in Erscheinung, insbesondere ist hier die Ulnarseite des Unterarms und der Hand befallen. Die Oberarmhaut ist weniger befallen, nur kleine teleangiektatische Inseln, teils langgestreckt, teils punktförmig, finden sich dort. Die Telangiektasien gehen an der Dorsalfläche der Finger bis zu 
ÜTher den nervösen Ursprung der telangicktatischen und anämischen Naevi. 237

den Endphalangen, dieselben ganz oder fast bedeckend. Der 3. und 5. Finger ist am meisten befallen, der Daumen fast völlig frej. Die Palmae manus sind beiderseits sehr stark verändert, durch dic Dicke der Haut tritt dies aber weniger in Erscheinung.

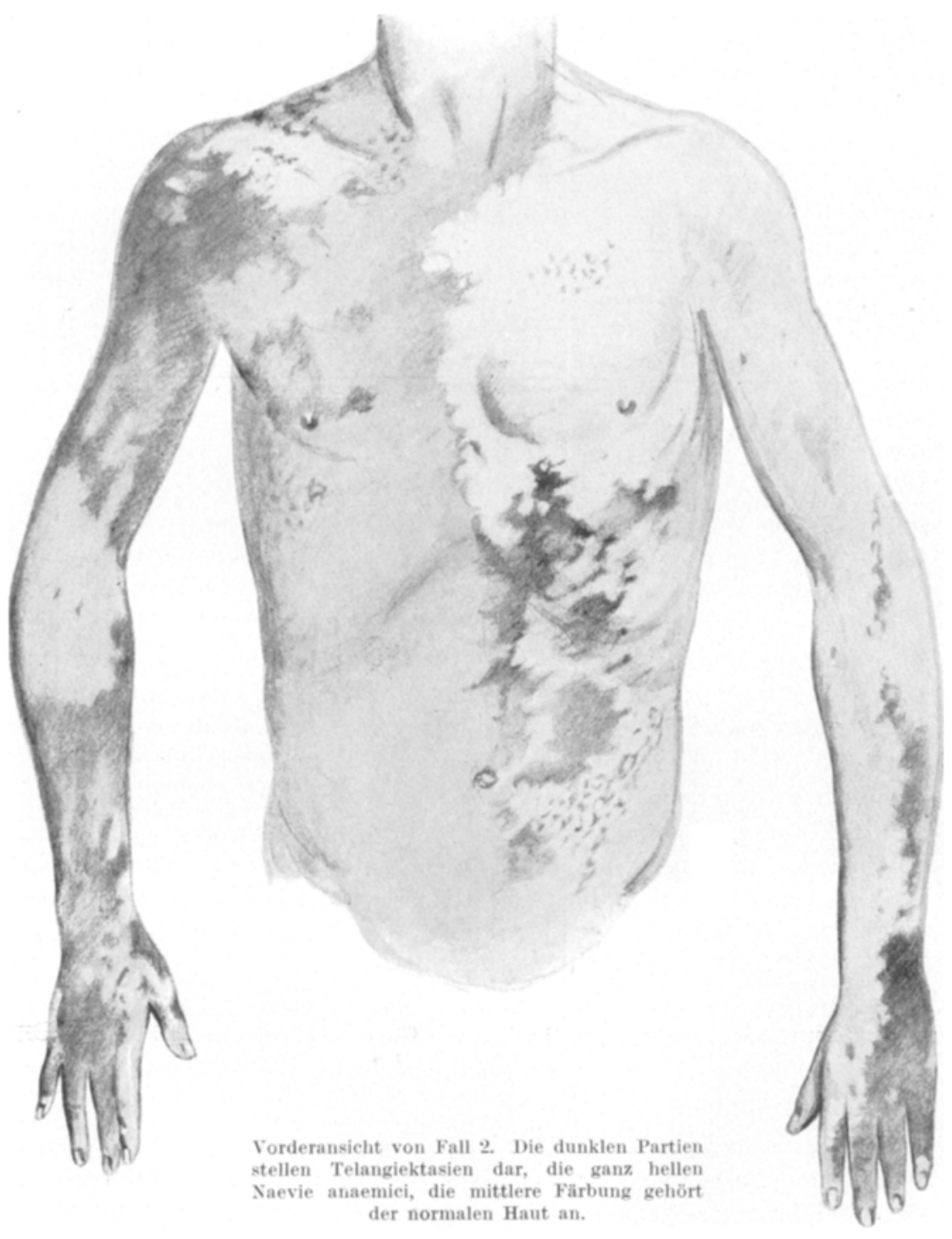

Das rechte Bein ist ganz frei, am linken finden sich an der Imnenfläche des linken Obersehenkels, ferner im Bereich der Patella, der inneren Knöchelgegend, des Fußrückens und der 1.-3. \%ehe teils strichartige, teils flächenhafte Telangiektasien. Wir sehen also, daß es sich um cinen über fast den ganzen Körper erstrecken- 
den, riesenhaften telangiektatischen Naevus handelt, der wesentlich an der Vorderund Rückenfläche des Rumpfes von kleineren und sehr großen unregelmäßigen anämischen Partien und normaler Haut durchzogen wird. Alle drei Formen von Hautpartien gehen, teils unvermittelt, teils ganz allmählich ineinander über, so daß wir also durcheinander gewürfelt haben:

Haut mit normaler Gefäßbildung,

Haut mit Hypoplasie in dem in der Einleitung angegebenen Sinne des Naevus anaemicus und starker Hyperplasie und Erweiterung der Hautgefäße.

Wenn ich nach der Schilderung dieser beiden ausgedehnten Naevi anaemici, die mit großen telangiektatischen Naevis kombiniert sind, auf meine einleitenden Bemerkungen zurückgreife, so habe ich dort hervorgehoben, daß für den einfachen Naevus anaemicus die Erklärung einer nervösen Entstehung durch Fehlen oder Unterbildung der Vasodilatatoren mit den Erscheinungen und Experimenten sich sehr gut in Einklang bringen läßt; denn alle diejenigen Reize, welche an der Gefäßmuskulatur selbst angreifen, sei es vorübergehender, sei es länger dauernder Art, können zu einer mehr oder weniger starken Füllung der Gefäße und einem Verschwinden des Naevus führen, dagegen Reize, welche durch Vermittlung des Nervensystems die Gefäße treffen und in der normalen Haut zu einer Gefäßdilatation führen, versagen im Naevus anaemicus. Voerner erörtert die Frage, in welchem Gebiet des Neurons die Störung wohl anzunehmen sei und kommt zu dem SchluB, daB wohl in den nervösen Apparaten der Gefäße selbst die Mißbildung liegen müsse, und es unwahrscheinlich sei, daß sie zentraler lokalisiert sei. Wenn wir nun diese Erklärung, welche alle Erscheinungen unserem Verständnis näherbringen, für den Naevus anaemicus akzeptieren, so entsteht die Frage, wie das gleichzeitige Vorhandensein kleinerer und vor allem größerer Telangiektasien zu erklären sei. Die enge Verbindung beider Formen, wie sie besonders unser letzter Fall aufweist, der Übergang beider ineinander, machen es doch wahrscheinlich, daß wir nicht verschiedene Ursachen für die Entstehung beider Naevusformen, wenn sie kombiniert sind, annehmen können - besonders bei der Abwesenheit sonstiger Mißbildungen — sondern daß wir eine prinzipielle Ursache für die beiden so diametral verschiedenen Naevusformen supponieren müssen, d. h. daß wir auch für die telangiektatischen Naevi eine Mißbildung der Gefäßnerven und zwar der Vasoconstrictoren annehmen müßten.

Nur eine Schwierigkeit ergibt sich bei diesem Erklärungsversuch: beim Naevus anaemicus handelt es sich um ein durch die Mißbildung der Gefäßnerven hervorgerufenes physiologisches Phänomen. Die Gefäße selbst sind in Größe und Zahl normal vorhanden, es besteht keine nachweisbare anatomische Veränderung. Bei den Telangiektasien dagegen handelt es sich nicht nur um stark erweiterte, sondern um zahlreiche neugebildete Gefäße. Läßt sich diese anatomische Ver- 
änderung mit der Mißbildung der Gefäßnerven erklären? Ich glaube, daß das möglich ist. Wir wissen aus der Zeit des völlig ausgebildeten Organismen, daß nach Unterbindung arterieller Gefäße ein Kollateralkreislauf mit Neubildung der Gefäße stattfindet.

Wir wissen ferner, daß, wenn bei entzündlichen Vorgängen die Gewebsformation in ihrer biologischen Beschaffenheit sich mehr dem embryonalen Zustande nähert, Neubildung von Gefäßen stattfindeu kann. Es ist deshalb nicht absurd, diese Erfahrungen auf die teleangiektatischen Naevi zu übertragen, besonders insoweit sie sich mit anämischen Naevis kombinieren. Wenn im fötalen Leben durch Fehlen der gefäßverengernden Nerven die neugebildeten Gefäße dauernd weit bleiben, infolgedessen ein stärkerer Blutzustrom statifindet, so kommen die befallenen Blutgefäße unter ähnliche Bedingungen, wie sie vorher für den voll entwickelten Organismus erwähnt wurden; und es ist begreiflich, daß besonders in dieser Zeit der Entwicklung des Organismus, in welcher das Proliferationsvermögen im fötalen Leben ein ungeheures ist, es nicht nur zu einer Erweiterung, sondern auch zu einer Neubildung von Gefäßen kommen kann. So würde also durch das Fehlen oder eine Unterbildung der Vasoconstrictoren sich die Entstehung der telangiektatischen Naevi erklären lassen. Daß bei den Naevusformationen dasselbe Prinzip in verschiedenen Gebieten durcheinander greift, ist uns ja sonst auch bekannt; und es würde unseren sonstigen Erfahrungen auch nicht widersprechen, daß, wenn wir für die vorliegende Naevuskombination, ausgehend von der Erklärung der anämischen Naevi, eine Mißbildung der Gefäßnerven als den wahrscheinlichen Grund überhaupt heranziehen, wir für die eine Naevusform, die anämische, eine Unterbildung der Vasodilatatoren, und für mit diesen kombinierten telangiektatischen eine Unterbildung der Vasoconstrictoren supponieren. Hieraus würde resultieren, daß sowohl der Naevus anaemieus, wie der mit diesem kombinierte Naevus telangiektaticus gewissermaß3en eine sekundäre Naevusform darstellt, welche lediglich die Folge einer primären, im Nervens ystem gelegenen Anomalie, eines primären Nervennaevus ist, so daß hiermit der Begriff eines primären und sekundären Naevus sich aus den vorliegenden Beobachtungen würde ableiten lassen. $\mathrm{Ob}$ wir berechtigt sind, auch für die isoliert vorkommenden telangiektatischen und sonstigen Gefäßnaevi diesen nervösen Entstehungsmodus anzunehmen, muß natürlich in suspenso bleiben. Ob hier überhaupt eine einheitliche Ursache anzunehmen ist, erscheint bei der verschiedenen Form und Lokalisation nicht wahrscheinlich. Immerhin läßt es sich nicht von der Hand weisen, daß ebenso, wie es sehr viele isolierte Naevi anaemici gibt, die nun doch mit größter Wahrscheinlichkeit als neurogen aufzufassen sind, auch isolierte Fenermäler in ähnlicher Weise entstanden sein können. Ich möchte 
erwähnen, daß ich bei 2 Fällen von Naevus telangiektaticus versucht habe, experimentell der Frage, ob hier die Vasoconstrictoren fehlen, näherzutreten. Ich injizierte bei 2 Fällen von reinen Telangiektasien an mehreren Stellen 1/2 cem Adrenalinlösung $1: 100000$, die ja in der normalen Haut durch Vasoconstriction Anämie erzeugt. Dieser Effekt blieb bei diesen Versuchen aus, während durch eine Erfrierung mit Aethylchlorid anscheinend eine Anämisierung stattfand, durch Tauchen in sehr kaltes Wasser die Naevi äußerlich eine mehr cyanotische Farbe annahmen.

Die Versuchszahl ist viel zu gering, um ein allgemeines Urteil zu ermöglichen, ich führe sie nur zur Ergänzung meiner obigen Ausführungen an, um zu weiteren Versuchen nach dieser Richtung anzuregen.

Historisch dürfte es nicht ohne Interesse sein, zu bemerken, daß3 bereits im Jahre 1872 Theodor Sim on ein Feuermal im Gesicht zurückführte auf eine intrauterine Mißbildung der vasomotorischen Nerven. Im großen und ganzen ist die nervöse Entstehung der Gefäßnaevi bis jetzt nicht nennenswert diskutiert worden; und ich glaube doch, daß gerade die geschilderten Kombinationen der anämischen und telangiek tatischen Naevi uns veranlassen müssen, in dieser Richtung die Gefäß naevi weiter zu erforschen, besonders auch nach der anatomischen Richtung, weil wir hierbei ja eventuell wichtige Aufschlüsse über die Verteilung der für die Hautpathologie so wichtigen Vasomotoren erhalten können.

Zum Schluß kann ich es mir schließlich doch nicht versagen, wenn es auch unwissenschaftlich erscheinen dürfte, auf den Volksglauben hinzuweisen, der besonders für die Feuermäler ein Versehen während der Gravidität heranzieht, d. h. als Ursache der Entstehung eine durch einen plötzlichen auf das Nervensystem erfolgenden Eindruck und durch Vermittlung des Nervensystems hervorgerufene Störung. Der Volksglaube ist ja doch manchmal der Wissenschaft etwas vorausgeeilt. Ich verweise nur auf die Beobachtung, da $B$ bei Bestehen vieler Warzen die Entfernung einer zum Versehwinden aller führt, eine Beobachtung, die ja durch einige gute Mitteilungen zuverlässiger Ärzte verifiziert worden ist. Nun, für das Versehen, welches im Volksglauben eine so große Rolle spielt, fehlen uns bislang irgendwelche beweisenden greifbaren Anhaltspunkte. Zurzeit muß man annehmen, daß die oft bizarre Form der Fenermäler die Phantasie des Volkes nach dieser Richtung hin gewissermaßen in Bewegung gesetzt hat. Immerhin ist diese ganze Frage des Versehens während der Schwangerschaft von ganz ernsthaften Forschern in den Bereich der Möglichkeit gezogen worden. Erst jüngst hat in einem eingehenden Aufsatz im Archiv für Eugenik Rohleder die Frage an der Hand der vorliegenden Literatur behandelt; und ich zitiere aus dieser seiner interessanten Arbeit nur, daß ganz ernsthafte 
Über den nervösen Ursprung der telangiektatischen und anämischen Naevi. 241

Forscher wie Ernst v. Baer, Johannes Müller, Bischoff, Lietzmann, Goethe u. a. sich mit dieser Frage befaßt haben. Etwas Greifbares und sicher Beweisendes liegt, soweit ich aus dieser Arbeit entnehmen kann, für den Volksglauben nicht vor. Rohleder glaubt, daß die Möglichkeit bestünde, daß es sich beim ,Versehen" der Graviden um eine Art psychischer Imprägnation handele, die dann durch Vermittlung des Nervensystems organische Veränderungen hervorbrächte, also etwas Ähnliches, wie in neuerer Zeit manche bei Hysterischen entstandenen organischen Hautläsionen, auch der Herpes zoster, von einigen Autoren gedeutet werden. Bislang aber muß man sagen, daß alle diese Erwägungen zurzeit noch bedeutend weniger wie Mutmaßungen sind; und ich führe diese Mitteilungen zunächst nur an, um alles zu erwähnen, was zur Hypothese der nervösen Entstehungsweise der anämischen und telangiektatischen Naevi irgendwie in Beziehung gebracht werden kann.

\section{Literatur.}

Theodor Simon, Über Nervennaevi. Dieses Archiv 1872. - Voerner, Über Naevus anämicus. Dieses Archiv. 1906. 82. - W. Fischer, Über Naevus anaemicus. Dieses Archiv 96. 1909. - Robert Stei n, Über Naevus anaemicus. Dieses Archiv 101. 1910. - E. Bru ner und A. B usch ke, Naevus anämicus. Ikonographia Dermatologica Fasc. VII. - Voer ner, Zur Kenntnis des Naevus anaemicus. 121. 1915. - Rohleder, Das „Versehen der Schwangeren“. Arch. f. Frauenk. u. Eug. 6. Heft 1-2. 